\title{
EL TALENTO: ANTECEDENTES, MODELOS, INDICADORES, CONDICIONAMIENTOS, ESTRATEGIAS Y PROCESO DE IDENTIFICACIÓN. UNA PROPUESTA DESDE LA UNIVERSIDAD CUBANA Y EL ENFOQUE HISTÓRICO-CULTURAL
}

\author{
Dalgys Pérez Luján, Dislayne González Morales y Yoel D Díaz Alfonso. \\ Centro Universitario José Martí Pérez, Facultad de Humanidades, Cuba
}

\section{INTRODUCCION}

Uno de los objetivos más importantes de la educación es promover las condiciones necesarias para que todos los alumnos alcancen el máximo desarrollo académico y personal a lo largo de su escolarización. Es justamente desde esta perspectiva de la educación, permeable a las distintas necesidades de los alumnos, que la atención de los alumnos talentos cobra especial relevancia.

Este grupo de alumnos resulta frecuentemente ignorado por el sistema educativo con la errónea convicción de que sus disponibilidades personales les hacen autosuficientes y por tanto, toda atención diferenciada es superflua e innecesaria. Al mismo tiempo prevalece la interpretación parcial y sesgada de la noción de alumno especial, entendiéndolo solo en el caso de los sujetos que por sus especiales circunstancias están en desventaja y tienen mayores dificultades para beneficiarse de la educación escolar.

Sin embargo contrariamente a lo que muchas veces se piensa, no resulta fácil organizar una enseñanza ajustada a sus necesidades ni ellos han vivido siempre la actividad de estudios como una actividad gratificante y con sentido.

Organizar la enseñanza para ajustarla a las necesidades de este tipo de alumnos ha de ser planificada, desarrollada y evaluada con rigor y en lo fundamental debe reflejarse en el Proyecto Educativo de los centros. Por estas razones es una actividad que precisa y genera procesos de colaboración entre los profesores, los alumnos y la institución.

La comunidad científica internacional cada vez presta mayor atención a esta problemática (Reis y Renzulli, 1985; Freeman, 1985; Dunn,R. y Dunn, K. 1992,1993; Arocas, E.; Martínez, P.; Samper, I. 1994; Alonso y Benito, 1996; Benito y Moro, 1997; Porter, 1999; Purcell y Renzulli, 1998 entre otros). En Cuba en los últimos años se han llevado a cabo diversas investigaciones orientadas a fundamentar e instrumentar alternativas para atender a este tipo de alumnos (Gallardo Flores, 2000; Lorenzo García, 1996; Córdova, 1996; Castro Guevara, 1997; Amechazurra Tam, 1999; Ávila Solís, 1999; Guerra Álvarez, T. 2001; Llivina Lavigne, 1999). Sin embargo estos trabajos han estado orientados fundamentalmente hacia la enseñanza primaria y media mientras en el sector universitario el estado actual del estudio del talento particularmente de la identificación revela déficit (Heller y Viek, 1999). 
Concretamente en nuestro país no existen experiencias fundamentadas ni sistematizadas en esta dirección anteriores al Proyecto de Investigación "La detección y estimulación del talento en la universidad" desarrollado por el Centro de Estudios de la Educación de nuestra universidad, del que forma parte esta investigación, y no se puede asegurar que las vías que se instrumentan para la detección del talento en el contexto universitario resulten efectivas.

Como problema psicológico el estudio del talento se considera con justeza uno de los de más difícil análisis. Esto se explica por las dificultades metodológicas de la investigación, condicionada por la complejidad de este objeto de estudio y por la necesidad de abordarlo en todas sus interrelaciones en las condiciones reales de vida y actividad. Partiendo de ello, hemos comprendido el talento como una estructura integral, tomado en sus complejas interrelaciones con la realidad y al enfocar nuestro estudio partimos de las ideas fundamentales de Mönks y Van Boxtel (1988) quienes incorporan el Modelo de los tres anillos de J. Renzulli (1978) que sitúa al talento en la intercepción de la creatividad, la inteligencia, la motivación y la dedicación en las tareas añadiendo al análisis las experiencias y los procesos de socialización del individuo. Así proponen el Modelo de la interdependencia triádica que incluye el contexto de la familia, los compañeros y la escuela. En nuestra opinión la clave de este análisis está en la categoría Situación Social de Desarrollo del eminente psicólogo L. S. Vigotsky. Tomando como referente esta perspectiva las interacciones mediatizan el proceso de interiorización de las formas superiores de desarrollo y a su vez los recursos personales del sujeto mediatizan las propias interacciones caracterizando su posición individualizada.

La educación especial de los estudiantes talentos en la universidad ayudará a formar el potencial humano altamente calificado que promueva el desarrollo económico social y cultural del país garantizando la formación de investigadores que busquen soluciones a problemas, que mejoren la educación y que impulsen la productividad.

\section{REFERENTES TEÓRICOS. APROXIMACION TEORICA AL PROBLEMA DEL TALENTO. ANTECEDENTES HISTORICOS SIGNIFICATIVOS EN EL ESTUDIO DE LA EXCEPCIONALIDAD INTELECTUAL}

\section{Un primer intento: el estudio de Galton}

El primer intento de análisis científico de la superdotación fue realizado por Galton (1869) quien no referencia directamente el concepto de superdotación sino de genialidad, es decir, trabajaba un concepto que se considera poco actualmente pero que resultaba muy significativo. Las implicaciones de esta noción son varias: manifiesta una clara diferencia de la normalidad casi en un sentido cualitativo, admite una serie de implicaciones culturales con connotaciones casi mitológicas y por último toma como referencia una forma de producción diferente a la normal pero socialmente valorada.

En sus trabajos, Galton $(1869,1874,1889)$ detectó una importante relación en lo que respecta a los antecedentes familiares, por lo que hace hincapié en la transmisión hereditaria del genio. Es necesario aclarar que su criterio esencial de validez fue la valoración social del rendimiento, estos dos elementos aportaron importantes sesgos en su trabajo que no realizó aportaciones importantes pero, en cambio, condicionó posteriores trabajos sobre el tema. 


\section{El macroestudio de Terman}

Otro de los científicos que resulta un referente importante en la historia de los estudios relacionados con esta temática es Terman quien en 1921 inició un proceso de identificación de escolares que mostraban un elevado coeficiente intelectual (C.I) en la versión de 1916 del Stanford - Binet desarrollada por él. El procedimiento consistía en aplicar la citada prueba a una serie de niños que habían sido seleccionados por sus maestros en función de los criterios de brillantez escolar y juventud, siendo considerado grupo experimental.

Esencialmente sus objetivos eran descriptivos, con un seguimiento longitudinal para controlar el desarrollo de los sujetos, sus características y aportaciones. Igual información se extrajo de un grupo de sujetos escogidos al azar el cual sería utilizado como grupo de control.

Algunas de las conclusiones que nos dejan ver su investigación son:

- Herencia y ambiente superior.

- $\quad$ Superioridad física.

- Puntuación en dos o tres años superior a la de los compañeros de clase en todos los tests de rendimiento.

- Mayor número de lecturas efectuadas.

- $\quad$ Nivel de intereses más elevados.

Los trabajos iniciados por Terman resultaron una tarea importante por los aportes que realizan. No obstante han recibido críticas bastante significativas. Genovard y Castelló (1990); Gowan (1979) coinciden en destacar como las más sustanciales las siguientes:

- La concepción de la inteligencia como una variable unidimensional.

- Falta de hipótesis explicativas para orientar el estudio.

- Grupo de control irregular en cuanto no resultaba suficientemente representativo de la población ni difería del experimental sólo en las variables implicadas en le estudio sino en muchas otras.

- Estructuración inadecuada del seguimiento longitudinal dado que no se controlaban adecuadamente las variables de cohorte y edad.

- Conocimiento por parte de los sujetos de que eran observados en el sentido de genios, influyendo esto en el auto-concepto $y$ en las expectativas y actuaciones de las familias $y$ profesores.

- Falta de homogeneidad en el C.I.

\section{La creatividad asociada a la excepcionalidad intelectual}

Uno de los saltos esenciales en el estudio de la excepcionalidad intelectual es la introducción de la creatividad como una variable relevante a partir del Modelo de la Estructura del Intelecto de Guilford (1967) en el cual aparecía con la denominación de producción divergente. Sin embargo, quien principalmente se 
especializó en esa materia fue Torrance. De hecho ahora es considerada una de las variables esenciales en la configuración de la superdotación.

A través del diseño del Torrance Test of Creative Thinking, Torrance intentó alcanzar la difícil tarea de medir la creatividad. Los resultados eran analizados a partir de varios índices:

- Fluencia

- Flexibilidad

- Originalidad

- Elaboración

Como se observa los índices que se tratan resultan muy susceptibles a la subjetividad y ambigüedad de la apreciación del investigador dado por el carácter general del test para medir la creatividad que al mismo tiempo resulta ser su mayor dificultad porque por su contenido no puede inscribirse en la motivación de ejecución esencial del sujeto. No obstante, su trabajo ha constituido una aportación importante desde la Psicología de la Excepcionalidad hacia la Psicología de la Inteligencia a partir de la consideración de esta variable y del intento de establecer sus índices.

\section{MODELOS DE LA EXCEPCIONALIDAD INTELECTUAL}

En la actualidad el tema de la excepcionalidad intelectual cobra vigencia, los estudios proliferan, se crean multitud de asociaciones y publicaciones especializadas y emergen múltiples modelos que vienen a confirmar que esta temática se ha ampliado acogiendo numerosos campos de la actividad humana (Jiménez Correa y Lou Royo, 1999). Izquierdo (1990) propone cuatro tipos de modelos a la hora de abordar el estudio de la excepcionalidad intelectual tomando en consideración el criterio que prima en las investigaciones de sus autores:

\section{Modelos basados en las capacidades}

Son aquellos que iniciaron el estudio del tema, dándole contenido al término. Entre los integrantes se incluyen Terman (1954), Taylor (1978), Gardner (1983) y la política misma de la U.S. Office of Education ( 1972, 1985). La excepcionalidad es abordada como la manifestación de un alto grado de talento que difieren en el nivel, factor, entre otros. Entre las ventajas que se le señalan a este modelo se encuentra la estabilidad en el tiempo, el temprano diagnóstico en los niños que favorece la intervención y como inconveniente la consideración de la excepcionalidad como una característica personal de origen innato.

\section{Modelos basados en el rendimiento.}

Estos exigen la demostración de las características y capacidades, eligiendo el rendimiento como criterio de validez. Como uno de sus principales exponentes se considera a Gagné (1985) cuyo modelo propone emplear el término superdotación para la competencia y talento en el ámbito del rendimiento y se esfuerza en demostrar cómo la superdotación se convierte en talento y cómo para ello precisa de catalizadores. Al no identificar la excepcionalidad como una identidad estable lo somete a un proceso de continuo seguimiento y a expensas de diversas interacciones. Como consecuencia, el criterio de rendimiento hay que aplicarlo con reservas en épocas tempranas. 


\section{Modelos de componentes cognitivos.}

Los teóricos cognitivos hacen mas hincapié en los procesos de orden superior y en las fases del procesamiento de la información que en el producto de excepcionalidad intelectual y en el empleo del término. Su atención está puesta en la elaboración de modelos y en el análisis de tareas. Entre los autores que aquí se incluyen se encuentran Borkowski (1986), Jackson y Butterfield (1986) y Sternberg (1977, 1981,1982).

\section{Modelos socioculturales.}

Los modelos socioculturales pretenden destacar que el sujeto excepcional es un producto de la sociedad en que vive, haciendo especial hincapié en el papel del contexto familiar y social en la potenciación o inhibición de ciertas conductas y habilidades. Los autores que responden a esta concepción son Tannenbaum (1983), Csikszntmihalyi y Robinson, Haensly, Reynolsy Nash Albert y Runco. Estas posiciones constituyen un reto para los defensores de los modelos basados en las capacidades porque cuestiona no solo la estabilidad de los condicionantes del rendimiento, sino también la estabilidad de las posibilidades de exigencias y criterios sociales del mismo (Izquierdo, 1990). Los modelos socioculturales demuestran con claridad la necesidad de una concordancia histórica favorable para poder llegar a un rendimiento excepcional.

\section{EL MODELO DE LOS TRES ANILLOS}

Hoy, tras varios años de estudio y experimentación la concepción más generalmente aceptada por los especialistas en el tema, es la de J.Renzulli, del Instituto de Investigación para la Educación de los alumnos Superdotados (Research Institute for Gifted children, University of Connecticut, USA, 1978), que propone el "modelo de los tres anillos o la puerta giratoria" que ha sido reconocido como una excelente contribución al esclarecimiento y comprensión de la configuración estructural psicológica de la superdotación humana (Genovard y Castelló, 1990; Jiménez Correa y Lou Royo, 1999).

Fig. 1. Modelo de los tres anillos.

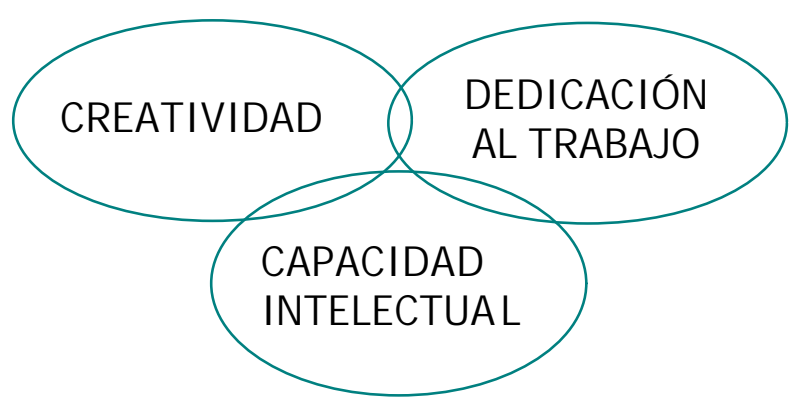

En la Fig. 1, entre los componentes que nos detalla esencialmente el modelo, Renzulli incluye también la capacidad intelectual superior a la media, que sigue entendiendo la inteligencia desde el rendimiento (como resultado y no como proceso) pero no constituye el criterio absoluto ni queda privilegiado en su modelo. La preponderancia que habitualmente se da a la capacidad intelectual en la definición de la superdotación queda modulada al hacerse referencia a otros dos criterios: la creatividad y los factores motivacionales; entendidos todos desde una perspectiva sistémica, que sin lugar a dudas amplía la 
posibilidad de identificación y de evaluación de la excepcionalidad intelectual (Pérez Luján y Álvarez Valdivia, 2002).

Genovard y Castelló (1990) manifiestan que esta combinación de aptitudes se ajusta a la relación existente entre la producción convergente (inteligencia general, lógica) y la producción divergente (creatividad).

Como se puede apreciar, el modelo de Renzulli enriquece considerablemente la descripción de las características de la superdotación, a pesar de ello desde este modelo las medidas continúan definiéndose cuantitativamente desde escalas que se construyen sobre la base de criterios comparativos y relativos a una población. Por ejemplo: capacidad intelectual superior a la media; alto grado de motivación y dedicación y creatividad elevada. Cuando se afirma que un alumno es superdotado o talentoso se hace referencia a que posee un nivel de rendimiento intelectual general o unas habilidades específicas excepcionales, tomando como punto de referencia al resto de los alumnos, que no están incluidos en la misma situación social de desarrollo que el alumno que está siendo evaluado (Pérez Luján y Álvarez Valdivia, 2002).

Lo que si queda claro es que el alumno superdotado presenta como principal diferencia respecto a otros, un sistema de tratamiento de información mucho más eficaz y productivo, y por tanto más susceptible de ser generalizado y aplicado a diferentes campos (Castelló, 1987). Expresado en estos términos continuamos haciendo referencia a constructos extraídos desde inferencias que se basan en comparaciones.

Como podemos ver, esta definición tiene sus limitaciones pero resulta interesante si tenemos en cuenta que garantiza la solidez teórica al proponer un modelo apriorístico claro, operativizando el campo de trabajo y definiendo las relaciones previstas, lo que orienta las investigaciones dirigidas a la conformación empírica del propio modelo por una parte y al desarrollo de instrumentos y procederes para la selección y la estimulación de alumnos con estas características, incluye la creatividad como una variable fundamental en la configuración de la superdotación e incorpora factores volitivos y motivacionales lo que implica un enfoque multilateral e integral en su estudio (Pérez Luján y Álvarez Valdivia, 2002).

Si bien el modelo de Renzulli se centra en el trabajo de las características de la superdotación, es conveniente realizar una distinción entre ésta y los talentos específicos. De este modo:

Superdotados: son alumnos que al presentar un nivel de rendimiento intelectual superior en una amplia gama de aptitudes y capacidades, aprenden con facilidad en cualquier área.

Talentosos: son alumnos que muestran habilidades específicas en áreas muy concretas.

Lo expuesto podría provocar ciertas interrogantes, pero de todas las posibles, hay una que merece especial aclaración. ¿Por qué asumir el modelo de Renzulli, si se centran en la superdotación y no en el talento? Pues porque no existen diferencias entre talento y superdotación, más allá de la que puede estar dada por la especificidad del talentoso y por el manejo más eficaz de la información que posee el superdotado de manera general; en esencia, se considera que en ambos deben existir la capacidad intelectual, la creatividad y la motivación y dedicación en las tareas como características indisolubles.

Si bien el modelo define operativamente la superdotación, para el profesor sería de mucha utilidad saber como se concretan en el funcionamiento de los alumnos estos criterios, lo que facilitará no sólo su identificación y evaluación, sino el diseño de estrategias educativas para estimular su desarrollo. Por tanto, 
sin descartar la real posibilidad instrumental que ofrece esta aproximación teórica, es conveniente que avancemos hacia la especificidad de la funcionalidad del desempeño intelectual excepcional. En este sentido Pérez Luján y Álvarez Valdivia (2002) señalaron como valiosos los trabajos sobre creatividad de A. Mitjans, el aprendizaje estratégico de Monereo y la voluntad de I. Peña Grass y Z. Nieves Achón, todos se valoran vinculados al perfil profesional que es, en definitiva, donde se expresa el talento buscado, sistematizándolos en la evaluación del talento vinculado a la profesión.

\section{INDICADORES DEL DESEMPEÑO CREATIVO DE LOS ALUMNOS CON EXCEPCIONALIDAD INTELECTUAL}

Se plantea que la capacidad creativa de los alumnos con excepcionalidad intelectual se expresa en la originalidad de muchas de sus realizaciones, las cuales resultan ingeniosas y poco comunes.

Una de las autoras que ha trabajado el tema de la creatividad, A. Mitjans (1989), plantea que es el proceso de descubrimiento o producción de algo nuevo que cumple las exigencias de una determinada situación social en la cual se expresa el vínculo de los aspectos cognitivos y afectivos de la personalidad.

Tomando como referente la concepción de personalidad que se sustenta en este enfoque, se coincide con los autores que reconocen que no es posible establecer un perfil de personalidad único, en base a rasgos, que caracterice a los individuos creativos. Realmente, la creatividad no es una cualidad general de la personalidad que se evidencia en todos los campos de acción del sujeto de forma generalizada, ni tampoco está dada por una sumatoria de rasgos y cualidades.

Es importante no considerar la creatividad como una habilidad más, sino como un complejo proceso de la subjetividad humana que tiene en su base un conjunto de recursos psicológicos que se configuran de forma específica y regulan el comportamiento creativo del sujeto.

Partiendo de los diferentes supuestos teóricos expresados hasta el momento ver que la creatividad es posible, entre otros actores, por la existencia en el sujeto de un conjunto de recursos de naturaleza cognitivo - afectiva que se configuran posibilitando la regulación de su comportamiento creativo. Ella es la expresión de la implicación de la personalidad en una esfera concreta de la actividad, el producto de la optimización de sus capacidades en relación con fuertes tendencias motivacionales donde el sujeto de la actividad está implicado como un todo.

Citando la tesis de la autora referenciada que define que el carácter personológico de la creatividad consiste en esencia en considerarla como expresión de la función reguladora, como expresión de configuraciones personológicas específicas que desempeñan un papel esencial en la determinación del funcionamiento creativo. Esta tesis es fundamentada por esta autora a partir de cinco elementos esenciales (A Mitjans, 1995 b: 19):

- En la creatividad se expresa el vínculo entre lo afectivo y lo cognitivo, que es la célula esencial de la regulación del comportamiento por la personalidad.

- La creatividad está asociada a la presencia de un conjunto diverso de elementos estructurales de la personalidad, entre los que se destacan las formaciones motivacionales complejas especialmente las intenciones profesionales y la autovaloración. 
- La comprensión de la creatividad está relacionada con la distinción conceptual entre las categorías sujeto y personalidad, lo que permite explicar la determinación psicológica de muchos comportamientos creativos.

- La creatividad es expresión de configuraciones personológicas específicas que constituyen variadas formas de expresión sistémicas y dinámicas de los elementos estructurales y funcionales de la personalidad, que intervienen en el comportamiento creativo.

El mayor aporte de los trabajos de esta autora, en opinión de Pérez Luján y Álvarez Valdivia (2002) se concreta en la formulación de los indicadores correspondientes a este proceso en términos de flexibilidad, originalidad, elaboración personal o reflexiva, independencia y estructuración temporal de los contenidos psicológicos, que aparecen explicados a continuación:

- Flexibilidad: Es la capacidad del sujeto para cambiar decisiones y proyectos y adecuarlos a nuevas exigencias, así como cambiar alternativas de comportamiento concretas. Permite a la persona reorganizar, reconceptualizar y revalorar los diferentes contenidos psicológicos de su personalidad.

- Originalidad: Capacidad para reflejar la realidad de manera particular creando formas nuevas, allí donde la personalidad se encuentra comprometida.

- Capacidad de autodeterminación o independencia: Autonomía relativa dentro del marco socio-histórico mostrando una actitud inconforme y realizando tareas por su propia iniciativa.

- Elaboración cognitiva personalizada: Elaboración reflexiva; redefiniciones con carácter constructivo-proyectivo, utilizando huellas experienciales y conocimientos adquiridos.

- Estructuración temporal de los contenidos psicológicos. Capacidad para organizar y estructurar los contenidos psicológicos en una dimensión futura, de tal forma que sean efectivos en el ejercicio de las funciones reguladoras de la personalidad.

- Perseverancia: Posibilidad de no abandonar las tareas a las que el individuo se enfrenta, por difíciles que sean los obstáculos para su culminación.

Tomando como referente los postulados de la autora citada se puede apreciar que el producto creativo es considerado entonces como el resultado de un proceso donde intervienen varios elementos, entre los cuales la personalidad en su función reguladora es esencial, porque, son los recursos personológicos que el sujeto posee y despliega en la consecución de sus principales objetivos, los que posibilitan el resultado creativo. Mientras más se implique el sujeto como personalidad en una esfera de actividad, mayores recursos personológicos utilizará y potencialmente mejores podrán ser sus realizaciones en esta área (Pérez Luján y Álvarez Valdivia, 2002). .

\section{INDICADORES DE LOS FACTORES MOTIVACIONALES Y VOLITIVOS}

Cuando analizamos la participación de los factores motivacionales en el desempeño intelectual, encontramos aportes importantes en los trabajos de J. Alonso Tapia (1999), donde queda demostrada ante todo la relación funcional que se establece entre la motivación de los alumnos por las tareas docentes y las 
pautas de actuación de los docentes. De manera que se impone una comprensión interaccionista de este elemento.

Sus estudios han hecho disímiles aportes entre los que se encuentran los indicadores para evaluar las diferencias motivacionales con que los alumnos acuden a las actividades docentes (Montero y Alonso Tapia, 1992). En concreto, los factores a que se hace referencia son: la disposición al esfuerzo, la motivación intrínseca, la ambición de las metas, la ansiedad facilitadora del rendimiento en contraste con el miedo al fracaso.

El alto grado de motivación y dedicación a las tareas docentes queda expresado en la posibilidad de disponer de gran cantidad de energía y de tiempo para realizar una actividad específica o a resolver un problema.

Se observa que el alto grado de motivación y dedicación a las tareas docentes se relaciona necesariamente con la voluntad. La regulación volitiva implica aquellos recursos personales que le permiten al sujeto una posición individualizada frente a las exigencias sociales en condiciones que resultan difíciles para él, mediante la adopción de decisiones y el planteamiento y consecución de objetivos conscientes con dimensión futura, que le posibiliten la regulación de su comportamiento presente (Z. Nieves Achón, 1998). Tal regulación consciente pose un carácter selectivo y es una manifestación de la personalidad, surge de ella y es guiado por ésta y, a su vez, fija su postura con respecto a los demás y con respecto a sí mismo, como sujeto que se autodetermina.

La sistematización de los criterios teóricos fundamentales y los resultados de las investigaciones preliminares realizadas sobre el desarrollo de la regulación volitiva del comportamiento, han revelado la importancia de tres indicadores funcionales en los cuales se centra el estudio de I. Peña Grass y Z.I. Nieves y que se explican a continuación:

- El planeamiento de metas.

- La toma de decisiones.

- El esfuerzo volitivo.

Los indicadores vistos tradicionalmente se concebían como eslabones, funciones o características del acto volitivo.

Analizando el primero de estos, se asume preferentemente el término meta; en tanto que desde el punto de vista teórico en la meta se expresa tanto la planeación por el sujeto de su comportamiento presente como la estructuración de su futuro, concretado en el intercondicionamiento de los objetivos y fines inmediatos y mediatos que la personalidad estructura como eslabón central de su autorregulación. (A. Konopkin, 1987; citado por Z.I. Nieves, 1998: 16).

Cuando se aborda la regulación volitiva resulta imprescindible valorar la relación entre las necesidades y los motivos a partir de la propia participación de la personalidad en la dirección de su comportamiento y en este sentido desempeña un papel fundamental el establecimiento de la meta como expresión de la intencionalidad motivada. 
El objetivo de este indicador, según las distintas posiciones teóricas que lo han trabajado, se revela en la medida en que el sujeto moviliza sus potencialidades hacia la obtención de la meta; lo cual presupone la implicación de la personalidad en calidad de metas propias.

Si analizamos la meta esta actúa como representación subjetiva que precede al comportamiento; pero sólo cuando el sujeto se moviliza en su consecución se convierte en meta real. (S.L.Rubinstein, 1967; citada por Z.I. Nieves, 1998: 19).

Se considera que el nivel de desarrollo del planteamiento de metas está determinado por las siguientes condiciones:

- Nivel de participación consciente de las tendencias motivacionales esenciales del sujeto en el planteamiento de sus metas.

- Grado de comprensión por el sujeto de las exigencias sociales.

- Valoración por el sujeto de sus posibilidades personales.

- Disposición del sujeto para asumir responsabilidad.

El segundo indicador referenciado revela el aspecto predominantemente movilizador de su conducta, de manera que su existencia se convierte en premisa del planteamiento de metas y éstas tienden a convertirse en metas propias en la medida en que en ellas se involucran conscientemente las tendencias motivacionales esenciales del sujeto sin obviar la estructuración de las relaciones necesarias con las exigencias sociales y las posibilidades reales del sujeto para alcanzarlas.

El planteamiento y la consecución de las metas, suelen implicar al sujeto en situaciones de elección, que involucran otro indicador volitivo, la toma de decisiones.

Este indicador abarca la existencia de varias alternativas para el sujeto en una situación de actuación, así como con su carácter plurimotivado, lo cual exige de él la selección definitiva de su variante de actuación y con frecuencia también implica la jerarquización de las motivaciones que lo orientan muchas veces después de un prolongado proceso de confrontación. La posición individualizada del sujeto al enfrentar las posibles alternativas de actuación y seleccionar finalmente su variante, asumiendo esta con la necesaria responsabilidad; constituye un indicador importante del desarrollo de los procesos de regulación y autorregulación de su personalidad.

Haciendo énfasis en este aspecto, podemos apreciar que la bma de decisiones, si bien se presenta con una dimensión temporal básicamente en el presente del sujeto, está asociada al futuro, en tanto las decisiones tomadas pueden afectar las metas mediatas. De igual forma el planteamiento de metas a veces implica tomar decisiones; existe una relación funcional entre estos dos indicadores volitivos.

Podemos entonces afirmar que el nivel de desarrollo de la toma de decisiones está determinado por las siguientes condiciones:

- Identificación por el sujeto de las posibles alternativas de actuación.

- Valoración por el sujeto de la información que posee acerca de cada una de las alternativas reconocidas. 
- Discernimiento por el sujeto de las ventajas y desventajas de las posibles elecciones o valoración de consecuencias.

- Confrontación y jerarquización por el sujeto de sus motivaciones esenciales.

- Selección de la variante de comportamiento de manera independiente.

- Disposición para asumir responsabilidad.

El esfuerzo volitivo constituye otro de los indicadores a abordar, ya que se considera uno de los medios indispensables de realización de la meta. Cuando el sujeto descubre la inadecuación de energía necesaria para alcanzar su objetivo y conscientemente se moviliza para comportarse activamente en correspondencia con los obstáculos que tiene que superar para llegar a la meta.

Para Peña Grass " el esfuerzo es indicador del nivel alcanzado cuando está vinculado a la economía de las energías, cuando se presenta no sólo para fortalecer o acelerar el proceso, sino para garantizar que la actividad volitiva ocurra como conscientemente se planteó, hasta el logro de su finalidad (I. Peña Grass,1989; citada por Z,I. Nieves, 1998: 25).

Se destaca en estos fragmentos de la autora el hecho de poder determinar el nivel de desarrollo volitivo analizando la capacidad del hombre de superar los obstáculos, esto es su capacidad para realizar el esfuerzo necesario y lograr la meta o tomar la decisión.

El esfuerzo volitivo expresa la tendencia del sujeto a estructurar conceptualmente sus conflictos, vivencias y estados, partiendo de la concientización y elaboración de los elementos internos y externos que afectan el comportamiento.

Deteniéndonos en el abordaje de este indicador podemos destacar que estos elementos se convierten en obstáculos para el sujeto, que sólo cuando es consciente de ellos y los enfrenta a través de una posición activa, puede condicionar su disposición positiva, implicando la autoestimulación como mecanismo psicológico fundamental.

Mediante nuestro abordaje por la teoría destacamos el nivel de desarrollo del esfuerzo volitivo como indicador a través de las siguientes condiciones:

- Identificación por el sujeto de la inadecuación de la energía para alcanzar la meta o tomar la decisión.

- Disposición consciente del sujeto para movilizar las fuerzas necesarias, en función de las motivaciones esenciales.

- La autoestimulación necesaria en el sujeto para operacionalizar en la situación que enfrenta y sus posibles cambios.

- Disposición consciente para supeditar al objetivo otras motivaciones que resultan importantes para el sujeto. 
Alcanza pues la regulación volitiva un nivel alto de desarrollo cuando en su comportamiento el sujeto es capaz de lograr que el planteamiento de metas, la toma de decisiones y el esfuerzo volitivo se correspondan favorablemente de manera plena. O sea, que de acuerdo con los grados de adecuación del funcionamiento de estos indicadores y cuán compatibles o no sean entre sí, podemos determinar los niveles de desarrollo de la regulación volitiva de la personalidad.

Los indicadores del desarrollo de la regulación volitiva del comportamiento son esencialmente el planteamiento de metas, entendida como la representación subjetiva de la finalidad del comportamiento, donde se involucran de manera dinámica, las tendencias motivacionales del sujeto, el grado de comprensión de las exigencias y la valoración de sus posibilidades personales para alcanzar la meta; la toma de decisiones, que se relaciona con la existencia de varias alternativas de comportamiento en una situación determinada, así como de su carácter motivado, lo que exige la valoración de las ventajas y desventajas de las posibles elecciones y la elección definitiva de su variante de actuación, lo que implica con frecuencia la jerarquización de las motivaciones que lo orientan, después de un prolongado proceso donde el sujeto en cuestión es capaz de confrontar y elaborar estos contenidos en función de sus intereses y motivaciones.

Hasta este momento hemos abordado el esfuerzo volitivo como un indicador importante al igual que los anteriormente planteados, de su definición y de los aspectos que tomamos como referencia para su análisis para la optimización de futuras investigaciones y de la nuestra en particular, pero no podemos observarlo en abstracto si no que seria importante analizar que se entiende por voluntad. La voluntad se define desde el esfuerzo volitivo, que se expresa en la posibilidad del sujeto para identificar la inadecuación de la energía suficiente para alcanzar la meta y/o tomar la decisión de manera que exista una disposición consciente de este para movilizar las fuerzas necesarias en función de las motivaciones esenciales, utilizando como recursos la autoestimulación consciente para operar en las situaciones que enfrenta y ante sus posibles cambios.

\section{LA ELEVADA CAPACIDAD INTELECTUAL DEL ALUMNO TALENTOSO. SUS INDICADORES}

La elevada capacidad intelectual es entendida como una capacidad de aprendizaje superior a la mayoría de los alumnos, pero no necesariamente como una inteligencia extraordinaria (E. Arocas, P. Martínez e I. Samper, 1994).

La capacidad de aprendizaje se relaciona con las estrategias cognitivas, que a través del tiempo, han ido ganando en relevancia al proporcionar una nueva vía teórica para atender a la diversidad (Monereo, $1990 ; 1993)$. Estas, representan modelos de variabilidad individual que nos permiten establecer diferencias entre las personas por el modo prevalente de percibir el medio, por sus procesos de información, por el modo de pensar, resolver problemas o actuar.

La concepción del aprendizaje escolar es introducida con una nueva óptica actual por C. Monereo y colaboradores, que en medida considerable coinciden con nuestra postura teórica y metodológica orientada a comprender el desempeño intelectual desde la perspectiva histórico cultural, con énfasis en la función autorreguladora de la personalidad. 
Mediante este estudio hemos asumido sus definiciones, en tanto en ellas se pone el acento en el cómo, en el modo prevalente que tiene el sujeto de conducirse, de operar mentalmente. El mismo ante una determinada tarea de estudio recurre a estrategias que le facilitan una ejecución exitosa, ya sea debido al aprendizaje, a la experiencia del medio o a la simple práctica. El modo en que un estudiante lleva a cabo las tareas del aprendizaje puede catalogarse como algo más que actuaciones azarosas ya que responden a modos de funcionamiento mental con cierta estabilidad, aunque modificables. La estrategia no es más que el modo, manera o forma preferente en que el sujeto percibe el medio, resuelve situaciones o actúa. El hecho de resaltar el carácter estratégico de los estilos cognitivos, acrecienta las posibilidades que brinda esta vía para la atención a la diversidad dentro del ámbito educativo (Pérez Luján y Álvarez Valdivia, 2002).

Las estrategias de aprendizaje del alumno se definen en calidad de «toma de decisiones, consciente e intencional, en la cual el alumno elige y activa de manera coordinada aquellos conocimientos declarativos y procedimentales que necesita para cumplimentar una determinada demanda, en función de las condiciones de la situación educativa en que se produce dicha demanda» (Monereo, Castelló, Clariana, Palma y Pérez Cabaní, en prensa; citados por M. Paneque, 1998).

- Únicamente podemos hablar de utilización de estrategias de aprendizaje cuando el estudiante da muestras de ajustarse continuamente a los cambios y variaciones que se van produciendo en el transcurso de la actividad, siempre con la finalidad última de alcanzar el objetivo perseguido del modo más eficaz que sea posible "(C. Monereo, 1998: 25). De esta forma, el estudiante minimiza el número de errores previos a la solución del problema asegurando que su respuesta sea la correcta después de un mínimo de tentativas.

"La utilización de estrategias requiere, por consiguiente, de algún sistema que controle continuamente el desarrollo de los acontecimientos y decida, cuando sea preciso, qué conocimientos declarativos hay que recuperar y cómo se deben coordinar para resolver cada nueva coyuntura "(C. Monereo, Ob. Cit.).

El perfeccionar de la estrategia está muy relacionado con la función reguladora de la metacognición, cuando se convierte en objeto consciente de la actividad del estudiante y está centrada en la estrategia; por lo que toda modificación en la misma es vista no únicamente en relación con los resultados a que conduce, sino vinculada a la serie de pasos o acciones que la conforman.

“ La regulación metacognitiva implica que, en algún momento de la solución del problema, el sujeto se cuestione acerca de lo que conoce hasta ese momento, qué le falta por conocer, cómo puede obtener el conocimiento que no posee. Es necesario que surjan cuestiones relativas a cómo la conducta seguida durante la solución ha estado relacionada con el alcance de los resultados obtenidos hasta ese momento " (A. Labarrere, 1996: 75).

A la luz de esta noción se admite que el estudiante que actúa estratégicamente debe ser, en alguna medida consciente de sus propósitos, y en función de estos, y de las características o condiciones de la situación en la que habrá de desenvolverse, elige y coordina la aplicación de uno o varios procedimientos de aprendizaje realizando acciones de control que conduzcan al perfeccionamiento de la estrategia. Con las acciones de control durante la solución, el sujeto puede cuestionarse acerca de lo inadecuado del instrumento que está aplicando sino también de sí mismo como sujeto de la actividad.

En opinión de Monereo, los indicadores que definen toda acción estratégica resultan:

- Conciencia: Actuar estratégicamente supone reflexionar sobre las consecuencias de una u otra opción. Una estrategia siempre deberá basarse en la actividad metacognitiva para reflexionar sobre la conducta a adoptar y su puesta en práctica aportará información relevante sobre los propios procesos mentales que favorecen el desarrollo metacognitivo. 
- Adaptabilidad: Dado que las condiciones de actuación donde tiene lugar la toma de decisiones varía durante el transcurso de la acción, el alumno deberá regular constantemente su comportamiento anticipando esas condiciones y planificando el curso de su actuación, reajustando el proceso y por último, evaluando y corrigiendo los resultados alcanzados en la misma.

Cuando se abordan algunos factores como posibles determinantes de un comportamiento estratégico, Monserrat Palma (1997), también argumenta la relación con los factores motivacionales. Compartiendo su criterio, se entiende que existe entre ambos procesos una relación bidireccional en la que, por un lado, el nivel motivacional como condición interna propiciará la intención del alumno y el esfuerzo correspondiente en el desempeño de alcanzar determinados objetivos mediante una actuación estratégica; y por otro, la evidencia de que el conocimiento por los alumnos de las estrategias que pueden utilizar y la disposición de los recursos adecuados para tomar decisiones respecto al proceso de aprendizaje creará expectativas positivas sobre el resultado de la actividad (Pérez Luján y Álvarez Valdivia, 2002).

En resumen, desde el abordaje de una visión novedosa de esta perspectiva del proceso de aprendizaje, de carácter intencional y propositivo, se concibe un aprendizaje flexible, capaz de transferirse a diferentes situaciones, dinámicas y variadas, en las que tiene lugar la actuación en el contexto socioeducativo no solo de los alumnos sino que incluye al profesor en un binomio que interactúan de forma eficaz e integrada.

Este comportamiento involucra las capacidades de anticipación, de planificación y de autorregulación durante la actividad intelectual, las cuales no se manifiestan al margen de los restantes factores descritos como determinantes del funcionamiento excepcional.

Es un hecho admitido que cualquier actividad cognitiva, incluyendo la aplicación de estrategias de aprendizaje, no puede observarse directamente, sino a través de vínculos indirectos como el lenguaje (explicar lo que ha pensado), y la conducta externa (acciones que dan cuenta de decisiones internas). Si ante determinada situación el alumno es capaz de poner en práctica estrategias cognitivas conscientes, entonces será capaz también de expresar lo que ha pensado por medio del lenguaje y realizará acciones externas que nos revelarán información acerca de las decisiones internas. Esto significa que el propio hecho de que el alumno sea capaz de formular y expresar verbalmente los procedimientos, da fe del carácter consciente de las decisiones que toma (Pérez Luján y Álvarez Valdivia, 2002).

La evaluación de todos estos indicadores en su interrelación dialéctica y sistemática, permite acercarnos sin dudas a la comprensión del funcionamiento excepcional de los alumnos talentosos.

Obsérvese, sin embargo, que los elementos hasta aquí citados son internos del sujeto y caracterizan su desarrollo actual. Realmente, como hemos venido señalando, la detección de los talentos resulta un proceso mucho más complejo que implica al ambiente y al potencial del alumno (Lewin y Michalson, citado por Freeman, J. 1988). Por tanto, terminaremos este análisis con la referencia a los factores externos que intervienen en el desarrollo del talento.

\section{CONDICIONAMIENTOS AMBIENTALES Y DESARROLLO PROXIMO DE LOS ALUMNOS TALENTOS}

Partiendo del reconocimiento de que el modelo de Renzulli constituye una ampliación dimensional importante y una corrección de las definiciones ya existentes, Monks y Van Boxtell (1988) argumentan en su 
contra que las características descritas tienen una naturaleza estática y no tienen suficientemente en cuenta las experiencias y los procesos de socialización, formulando un modelo de la interdependencia triádica. Estos autores proponen incluir los marcos sociales específicos de la escuela, los compañeros y la familia.

Fig. 2. Modelo de la interdependencia triádica, Monks y Van Boxtell (1988).

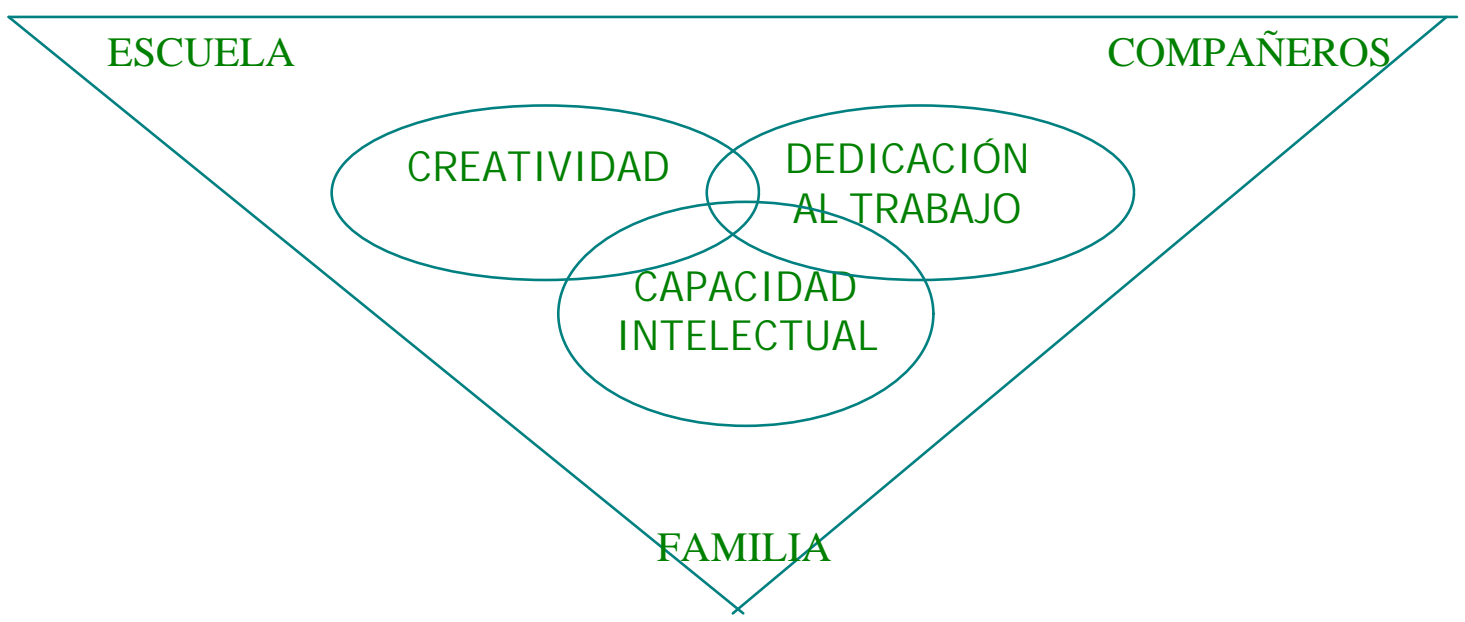

Cada vez es más frecuente que los autores que han trabajado el tema tomen en consideración los factores relacionados con el contexto. En este sentido tenemos que subrayar que el talento no es resultante ni de particularidades internas al sujeto ni de las influencias del medio vistas de manera aislada. Adentrarnos en el tema del talento desde esta perspectiva supone adscribirse a los postulados del enfoque Histórico Cultural (Pérez Luján y Álvarez Valdivia, 2002). El concepto situación social del desarrollo introducido por Vigotsky y desarrollado por L. Bozhovich, resulta esclarecedor de la influencia que ejercen cada uno en el desarrollo psíquico. Por este término Vigotsky (Ctdo. por Bozhovich, 1973), designó aquella combinación especial de procesos internos del desarrollo y de las condiciones externas que condiciona la dinámica del desarrollo psíquico y las nuevas formaciones psicológicas cualitativamente peculiares.

Actualmente se está reconociendo de forma general que es poco probable que los alumnos talentosos alcancen su potencial a menos que posean los medios psicológicos y físicos para desarrollarlos; esto implica que los recursos para el desarrollo de cualquier habilidad especial son, en sí mismos, de alguna forma parte del talento (Freeman, J. 1988). Pero el contexto es mucho más que el simple escenario de la ejecución de los productos de la mente o de la recepción de información, es parte esencial de la propia trama de la actividad psicológica.

Las condiciones externas constituyen un sistema único cuyo centro son las influencias vinculadas a la posición que el superdotado ocupa entre los que los rodean y son significativas si se concretan, como ha quedado explícito en el modelo de Mönks y Van Boxtel, en las exigencias del maestro; las de la familia y las de sus compañeros de clase.

Como puede apreciarse a partir de nuestro abordaje, la identificación del talento incluye necesariamente el establecimiento de un diagnóstico de su contexto educativo, familiar y grupal: ¿qué es?, ¿qué siente?, ¿qué quiere?, ¿qué hace? y en qué ámbitos, escenarios, grupos, dentro de qué sistemas de actividad y cómo se articulan todos ellos. 
El énfasis en el cómo se articulan se explicita en el concepto de situación social de desarrollo, en la combinación especial de las condiciones externas y de los factores internos, pues las condiciones del medio no siempre ejercen la misma influencia sobre el desarrollo, varían en dependencia de las propiedades psicológicas formadas anteriormente a través de la cual se mediatizan.

Podemos afirmar entonces que si queremos comprender como influye el medio en el talento es necesario analizar en que relación con sus necesidades se encuentra este medio, en que medida es capaz de satisfacerlas o no.

A su vez, esta concepción del desarrollo psíquico desde la obra de Vigotsky nos convida a tomar conciencia en la valoración de otro factor importante que está contenido en la afirmación de que las funciones humanas, en ese desarrollo cultural, aparecen dos veces: una vez en el plano social, como función compartida entre dos personas (el que aprende y el que enseña); como función interpsicológica y una segunda vez, en el plano psicológico, como función de un sólo individuo, como función intrapsicológica. La conversión de lo inter en intrapsicológico se da a través del mecanismo de la interiorización del proceso psíquico que implica la transformación de la estructura de la función, la constitución de una nueva construcción.

Al respecto Vigotsky señala que "toda la historia del desarrollo psíquico del niño nos enseña que desde los primeros días de vida, su adaptación se logra por medios sociales a través de las personas circundantes. El camino que va de la cosa al niño y del niño a la cosa pasa a través de otra persona . (Vigotsky, 1987)). Tomando en consideración este referencial teórico, el carácter inicialmente exterior social de las funciones psíquicas y la importancia decisiva de la actividad conjunta en el proceso que lleva a su interiorización, resulta pertinente introducir el concepto de zona de desarrollo próximo para una óptima comprensión del origen y desarrollo del talento. La zona de desarrollo próximo que determina la esfera de los pasajes del sujeto de lo que puede hacer solo a lo que es capaz de realizar con otro (con responsabilidad diferencial en cuanto a sus dominios simbólicos), es considerado como el momento decisivo en las interrelaciones del desarrollo y la enseñanza. Lo central es estudiar la posibilidad de que el alumno se eleve, mediante la actividad conjunta, a un nivel superior, pasando de lo que sabe hacer a aquello que aún no puede hacer sólo.

Desde este presupuesto lograr la eficiencia para la identificación del talento incluye crear y utilizar las zonas de desarrollo próximo. Esto implica que el maestro se ocupará en su diagnóstico no solo de los conocimientos y conductas efectivas sino de las conductas o conocimientos en proceso de cambio (Pérez Luján, 2000).

En esta pesquisa resulta esencial la información procedente de las interrelaciones talento- maestro en el proceso de ejecución durante el planteamiento y solución de las tareas diagnósticas. O sea, la investigación no se puede limitar al establecimiento del nivel de desarrollo actual del supuestamente talentoso, que constituye el umbral inferior indispensable para la enseñanza mediante las tareas que puede realizar de forma autónoma. Este nivel resulta insuficiente para determinar el estado de su desarrollo. Hay que considerar además el umbral superior conformado por aquellas funciones en proceso de maduración que nos indican por un lado el potencial del alumno y por otro las necesidades educativas que condicionarán las exigencias del medio hacia él, facilitándole externamente los mediadores para la interiorización durante el proceso educativo (Pérez Luján, 2000). 
Hasta lo que hemos abordado podemos plantear que en general, se suele tomar la zona de desarrollo próximo como el constructo individual sin considerar que la zona de desarrollo próximo de un sujeto es una función del contexto en que vive: la investigación del contexto converge así totalmente con la del desarrollo.

La necesidad de contar con una interfaz externa e interna a la vez, individual y social, en que se produzca la distribución de las funciones psicológicas o del procesamiento, abre así el camino a un cambio conceptual profundo tanto para la idea de contexto como para la de desarrollo (Del Río y Álvarez, 1994).

\section{EL PROCESO DE IDENTIFICACION}

Según Genovard y Castelló (1990), al hablar de identificación hacemos referencia a un conjunto de procedimientos que podrían agruparse en dos categorías básicas: procedimientos relacionados con la detección de los alumnos talentosos y procedimientos relacionados con la medida de sus capacidades y destrezas individualmente. En el primero de los casos, nos acerca al tratamiento de un enfoque diferenciado en el cual el principal objetivo es discriminar entre los sujetos con capacidad superior y los sujetos normales. El segundo punto, la medida de las capacidades y destrezas, es individualizado, refiriéndose estrictamente a la evaluación del perfil del sujeto excepcional (Alvino, 1985; citado por A. Castelló y C. Genovard, 1990: 107).

Pero muchos se preguntarán cual es el objetivo principal de esta etapa de identificación y que aclaramos a continuación. El objetivo de la identificación de los alumnos talentosos es poder anticipar y planificar mejor el trabajo para ajustar la preparación de manera que puedan conseguir el desarrollo más completo de sus potencialidades. Si la escuela debe ofrecer una respuesta educativa ajustada a las necesidades del alumnado, la escuela también debe estar preparada para identificar a los destinatarios de ayudas especiales.

En nuestro marco investigativo, el contexto universitario, donde lo que se persigue es la educación superior de los estudiantes, esto desde a posición que hemos asumido en nuestra investigación, debe formar parte de las actividades de planificación y organización, a fin de discriminar qué necesidades educativas diferenciadas presenta cada estudiante. La plena realización de este proceso permite, sin dudas, diseñar y planificar la respuesta educativa más adecuada, tanto como superar la mera labor de instrucción.

No pretendemos presentar la tarea de identificación de los estudiantes muy capaces, como u "trabajo extra", o como más trabajo; sino que más bien se considere que la actividad diaria en el aula debe enfocarse en este sentido, ya que la identificación, más que una tarea que se efectúa en un momento concreto, debe contemplarse como un proceso en que se analicen cuidadosamente distintas observaciones sobre comportamientos y realizaciones de los alumnos.

\section{LAS ESTRATEGIAS DE IDENTIFICACION}

Las principales estrategias de identificación, según Genovard y Castelló (1990: 110), serían las siguientes: 


\section{Identificación basada en medidas informales.}

En la primera fase de este tipo de estrategia se utilizan cuestionarios o autoinformes, para pasar a las medidas individuales y formales en la segunda.

La principal ventaja de este planteamiento es que se consigue una gran economía de tiempo y esfuerzo en la primera identificación, además de ciertos indicios sobre el perfil excepcional del sujeto (a través de la información que pueden brindar los profesores y otros estudiantes) que pueden también facilitar la segunda fase del procedimiento (medida o evaluación de las capacidades y destrezas del sujeto excepcional).

Como hemos hecho referencia, estos puntos favorables dependen muy claramente de los instrumentos informales. Suele ser bastante factible que existan distorsiones y sesgos en las respuestas a los mismos, tales como: comprensión de los enunciados o de algún término implicado, precisión de las definiciones, entre otros. Por otra parte, dado que su principal ventaja es la economía de tiempo, es necesario que sean forzosamente limitados, o sea, de poca extensión (A. Castelló y C. Genovard, 1990: 111).

Podemos entonces arribar a una conclusión muy importante respecto a este tipo de estrategia, y es que ella, puede considerarse la más adecuada si se dispone de medidas informales debidamente ajustadas al tipo de población con que se trabaja, lo que implica que se deberá emitir en la validación y fiabilización sistemática de dichos instrumentos.

\section{Identificación basada en medidas formales.}

Esta modalidad, como su nombre lo indica, difiere de la anterior en que el proceso de identificación se inicia ya con medidas de tipo formal, por lo que se evalúan directamente los distintos componentes implicados en la excepcionalidad. La aplicación de esta estrategia se lleva a cabo sobre toda la población, es decir, no se conoce aún qué sujetos son supuestamente excepcionales. En este caso se suele prescindir de las medidas contestadas por otras personas (padres, maestros, compañeros), dado que no tienen mayor utilidad. Además, la segunda fase de la identificación se reduce enormemente, pues la parte de la información ya ha sido obtenida en la primera fase.

Las conclusiones a las que nos permite arribar esta segunda estrategia sobre sus ventajas es que de este método se originan en el acceso directo a las variables, por lo que se dispone de pruebas desde un primer momento y no de indicios por confirmar. También, reduce el ámbito de aplicación y, como ya se ha comentado, de la segunda fase del procedimiento.

A pesar de sus ventajas, en cambio, resulta una estrategia muy costosa en la primera fase, puesto que los instrumentos formales son normalmente largos de aplicar y las condiciones de aplicación resultan mucho más rígidas.

Desde esta perspectiva podemos asegurar que la cantidad de tiempo y esfuerzo que se necesita movilizar es mucho mayor que en la anterior estrategia, dado que en este caso se deben utilizar muchos más instrumentos (no se dispone de ningún indicio con respecto a la forma de excepcionalidad de los sujetos) y la cantidad de sujetos es mucho mayor.

Esta estrategia es desaconsejable, a no ser que no se disponga de instrumentos informales debidamente validados y fiabilizados (A. Castelló y C. Genovard, 1990: 112) 


\section{Análisis individualizados.}

Este tipo de procedimiento se aleja en alguna medida de los estudios más experimentales o poblacionales y se centra en el análisis de las características específicas de los sujetos, abarcando los datos conjuntos de los dos apartados anteriores y, además, obtienen informaciones de tipo biográfico. Haciendo generalizaciones sobre este aspecto estaremos de acuerdo en que esta estrategia sólo se utiliza en investigaciones muy específicas cuyo objetivo se centra en los aspectos que han propiciado cierto tipo de excepcionalidad.

Resulta evidente cuán costosa llega a ser, a pesar de que se nos presenta como un nivel heurístico importante. A su vez, es la única forma de acceso al trabajo con sujetos excepcionales fuera del contexto psicopedagógico o de investigación poblacional.

En el acercamiento por las estrategias que proponen los autores y que nosotros por su utilidad hemos decidido interpretar y abordar, estos tres procedimientos constituyen las estrategias empleadas para los procesos de identificación. En cualquiera de los casos, estas estrategias buscan la diferenciación de la normalidad, bien por la vía de la alta habilidad en un componente específico o bien por la de competencia en múltiples componentes.

Resulta importante destacar que en la presente investigación hemos asumido la primera de las estrategias descritas anteriormente, es decir, la Identificación basada en medidas informales, teniendo en cuenta que constituye la variante más ventajosa dentro del proceso por las posibilidades que nos ofrece.

\section{CONCLUSIONES}

La estrategia psicopedagógica para la identificación y evaluación de estudiantes universitarios talentos debe inscribirse en el ámbito de la propia formación profesional, a partir de la relación entre las demandas académicas profesionales y las necesidades y posibilidades de los estudiantes para responder a ellas desde su desempeño.

La efectividad en la detección del talento supone contemplar múltiples vías de acceso (diferentes procedimientos, diversas fuentes, diversos contextos), usadas de forma complementaria, dado que pueden no manifestarse en alguna de ellas. La forma concreta de ponerla en práctica atendiendo en la primera fase a los criterios de profesores, de otros estudiantes, de los propiamente seleccionados y en la segunda a la evaluación de estos últimos, permite realizar una valoración objetiva y ajustada al contexto socioeducativo.

Un proceso de selección discriminativa eficiente con una concepción más abarcadora debe incluir indicadores como: el aprendizaje estratégico, la creatividad, la voluntad y el análisis del contexto donde debe considerarse como índice la relación profesional con otros significativos y el carácter de la tarea en función de las necesidades de creación, cognitiva y de las exigencias profesionales en su desarrollo.

Tomar como pilar el enfoque histórico-cultural implica proponer un camino metodológico acorde a sus presupuestos teóricos. Este enfoque da frente a las diferentes concepciones mecanicistas y dualistas de la relación del sujeto con su contexto desarrollador, es decir, se asume una concepción sistémica donde el mismo sea protagonista de sus áreas de actuación. 
Las situaciones diagnósticas por sus exigencias deben facilitar la expresión de los comportamientos comprometidos con el talento y de las relaciones intersubjetivas significativas para el sujeto que a su vez permitan crear y utilizar las zonas de desarrollo próximo.

\section{BIBLIOGRAFIA}

ALONSO TAPIA; J. (1992): Motivación y aprendizaje en el aula. Cómo enseñar a pensar. Madrid. Aula XXI, Santillana.

ALONSO TAPIA; J. (1999): Efectos motivacionales de las actividades docentes en función de las motivaciones de los alumnos. En Monereo, C (coord). El aprendizaje estratégico: enseñar a aprender desde el currículo. Aula XXI, Santillana.

ALONSO, J.A.; Benito, y. Superdotados: adaptación escolar y social en secundaria. Madrid: Narcea, cop. 1996. 285p (Secundaria para todos) ISBN 84-277-1101-8 [UAB UB UdL UPC Uvic]

ÁlvareZ VALDIVIA, I. M. Investigación cualitativa. Diseños humanísticos. Material de estudio. F.C.S.H U.C.L.V.

AmeChAZURRA TAM, O.L. (1999) Una Propuesta didáctica para la estimulación del desarrollo intelectual de los preescolares. Tesis en opción al grado de Doctor en Ciencias Pedagógicas. Instituto Central de Ciencias Pedagógicas. La Habana.

ANTONIJEVIC, N. y CHADWICK, C. (1993). Estrategias cognitivas y metacognitivas en la educación del futuro. Cinterplan: Caracas.

Arocas SÁnchez, E.; MARTínez Coves, P. y SAmper CAyuelas, I. (1994) La respuesta educativa a los alumnos superdotados y/o con talentos específicos. Valencia: Ministerio de Educación y Ciencias.

ÁvILA Solís, M. A. (1999) La Batería de ejercicios físicos CRIEF: una innovación para detectar talentos deportivos. Tesis en opción al grado de Master en Investigación Educativa. Instituto Central de Ciencias Pedagógicas. La Habana.

BeLtRÁN J. (1993) Procesos, estrategias y técnicas de aprendizaje Madrid: Síntesis.

BERgan, J. R. y DUNN, J.A. (1980) El desarrollo de la creatividad. En: Psicología Educativa. México: LIMUSA NORIEGA.

BERNARD, J.A. (1993). Estrategias de aprendizaje y enseñanza: evaluación de una actividad compartida en la escuela. En C. Monereo (Ed.), Las estrategias de aprendizaje Procesos, contenidos e interacción Barcelona: Domenech.

BIGGS, J.B. (1979) Individual differenees in study processes. British Journal of Educational Psichology, 48, 266-279.

BOzHOVICH, L. (1976): La personalidad y su formación en la edad infantil. Pueblo y Educación.

Del Río P. Y Álvarez A. (1994): "Ulises vuelve a casa: Retomando al espacio del problema en el estudio del desarrollo". Infancia y aprendizaje, 66, 21-45. 
CASTElló, A. (1987): La integración escolar del alumno excepcionalmente dotado. En C. Monereo (ed.): Áreas de intervención del psicólogo de la educación en la integración escolar del alumno con necesidades educativas especiales. Badalona: Federación ECOM.

CASTRO GueVARA, O.L. (1997) Una estrategia para el desarrollo del comportamiento inteligente en niños con discapacidad visual. Tesis en opción al grado de Master en Ciencias Pedagógicas. Instituto Superior Pedagógico. Ciudad de La Habana.

Coll, C., Martin, E., Mauri, T. Miras, M., OnRubia, J., SolÉ, I. y ZABAla, A. (1993) El constructivismo en el aula Barcelona. Grao.

CóRDOVA LLORCA, M.D. (1996) La Estimulación intelectual en situaciones de aprendizaje. Tesis en opción al grado de Doctor en Ciencias Pedagógicas. Instituto Superior Pedagógico. Ciudad de La Habana.

DUNN, R. y DUNN, K. (1993). Teaching secondary students through their individual learning styles: Practical approaches for grades 7-12. Boston: Allyn and Bacon.

ENTWISTLE, N.J. (1979) Approaches to learning and levels of understanding British Educational Research Journal, 1,514 .

FELDHUESEN, J. F. (1985): The teacher of gifted student. Gifted Educational International, 3, 87-93.

FREEMAN J. (Ed. 1985). Los niños superdotados. Aspectos psicológicos y pedagógicos. Madrid: Santillana.

FREEMAN, J. (1988): Los niños superdotados. Aspectos pedagógicos y psicológicos. Madrid. Santillana.

GAGNÉ, F.(1985): Giftenes and talent: Reexancining and reexamination of the definition. Gifted Child Quaterly, 29, 103-112.

GALLARDO FloRES, P. (2000) La Importancia de la ayuda externa en el desarrollo del sujeto talentoso. Tesis en opción al grado de Master en Investigación Educativa. Instituto Central de Ciencias Pedagógicas. La Habana.

García, E y Pascual, F. (1994) Estilos de aprendizaje y cognitivos. En A. Puente (Ed), Estilos de aprendizaje y enseñanza. Madrid. CEPE.

Genovard Roselló, C. y CAstelló TaRrida, A. (1990) El límite superior: aspectos psicopedagógicos de la excepcionalidad intelectual. Madrid: Pirámide.

GENOVARD, C (1990). Las estrategias de aprendizaje desde la perspectiva de la Psicología de la Instrucción. En C. Monereo (Comp.). Enseñar a aprender y a pensar en la escuela. Madrid: Visor

GENOVARD, C. (1983): "Educación especial para profesores de educación especial de niños excepcionales superdotados: inventando el futuro"; Educar, 3, 27 - 46.

GONZÁLEZ ReY, F. (1985): Psicología de la personalidad. Pueblo y Educación.

GonZÁlez Rey, F.; MitJAns A. (1989): La personalidad: su educación y desarrollo. La Habana: Pueblo y Educación.

GonZÁlez ReY, F. (1987). Motivación profesional en adolescentes y jóvenes. La Habana: Pueblo y Educación. 
GONZÁLEZ SERRA, D. (1976). Criterios y métodos para el estudio de la motivación. La Habana: Editora Universitaria.

GowAN, J. (eds) (1979): Educating the ablest. Peacock, Itasca, Illinois.

Guilford, J. P. (1967): The nature of human intelligence. New York: Mc Grow Hill.

HELLER, K.A. y VIEK, P. (1999) Support for university students: Individual and social factors. En: Grayson, P (Ed). (1999) The performance of gifted high school students in university. Toronto: Institute for Social Research, York University.

IZQUIERDO MARTíneZ, A. (1990): La superdotación. Modelos, estrategias e instrumentos para su identificación. Madrid: Universidad Complutense.

JiMÉnEZ CorREA, A. y Lou Royo, M. A. (1999): Necesidades educativas del niño superdotado. En M. A. Lou Royo y N. López Urquízar. Bases psicopedagógicas de la Educación Especial. Madrid: Pirámide.

KATHENA, J. (1982): Educational Psychology of the gifted. New York: John Willey and sons.

Kolb, D (1984). Experimental learning Experience as the source of learning and development Englewood. Cliffs. Prentice-Hall.

LabARRere SarduY, A. F. (1996) Pensamiento. Análisis y autorregulación de la actividad cognoscitiva de los alumnos. La Habana: Pueblo y Educación.

LLIVINA LAVIGNE, M.J. (1999) Una Propuesta metodológica para contribuir al desarrollo de la capacidad para resolver problemas matemáticos. Tesis en opción al grado de Doctor en Ciencias Pedagógicas. Instituto Superior Pedagógico. Ciudad de La Habana.

LÓPEZ GARCíA, J.M y VICENT PARDO, M.J. (1997). La LOGSE y el desarrollo del currículo en la Educación Secundaria. Madrid Colegio Oficial de Biólogos.

LORENZO GARCíA, R. (1996) El Talento en a escuela primaria. Tesis en opción al grado de Doctor en Ciencias Pedagógicas. Instituto Superior Pedagógico. Ciudad de La Habana.

MARTON, F. (1984). The experience of learning Edinbourgh: Scottish Academic Press.

Mitjans MARTíneZ, A. (1995a) Creatividad, Personalidad y Educación. La Habana: Pueblo y Educación.

MitJANS MARTínEZ, A. (1995b) La escuela y el desarrollo de la creatividad. Revista Educación, no 85 (mayoagosto)/Segunda Epoca, 18-24

MONEREO, C. (Comp.)(1990). Enseñar a aprender y a pensar en la escuela. Madrid: Visor.

MONEREO, C. (Comp.)(1993). Las estrategias de aprendizaje. Procesos, contenidos e interacción. Barcelona: Domenech.

Monereo, C. (coordinador); Castelló, M.; Clariana, M.; Palma, M. y Pérez Cabaní, M. L. (1998) Estrategias de enseñanza y aprendizaje. Formación del profesorado y aplicación en el aula. México: Biblioteca del Nominalista.

MONKS, F.; Van Boxtel, H.W.(1988): Los adolescentes superdotados: una perspectiva evolutiva. En J. Freeman (ed.) Los niños superdotados. Aspectos psicológicos y pedagógicos. Madrid: Santillaana Aula XXI. 
MONTERO, I. y ALONSO TAPIA. (1992): El cuestionario MAPE - II , en J. Alonso Tapia,J. Motivar en la adolescencia: Teoría, evaluación e intervención, pp. 263-280, Madrid, Servicio de Publicaciones de la Universidad Autónoma.

Morenza Padilla, L. y TerRé CAMACHO, O. (1998) Escuela Histórico-cultural. Revista Educación, no 93 (enero-abril)/Segunda Epoca, 2-11.

NeWLAND, T. W. (1976): The gifted socioeducational perspective. Nueva Jersey: PrenticeHall, Englewood Cliffs.

NiEVES ACHÓN, Z. I. (1998) Entrenamiento sociopsicológico para estimular el desarrollo volitivo en jóvenes. Tesis en opción al grado de Doctor en Ciencias Psicológicas. Santa Clara.

PÉREZ LUJÁN, D. (2000). Estrategia psicopedagógica para la detección del talento en la universidad. Tesis en opción al grado de Master en Ciencias de la Educación. Santa Clara.

PÉREZ LUJÁN, D. y ÁlvaReZ VALDiviA, I. (2002). La comprensión y el desarrollo de la excepcionalidad intelectual. Necesidad de trascender del enfoque centrado en el sujeto al análisis funcional y de contexto. Aula Abierta. №.79. Junio. Universidad de Oviedo, 18-23.

PRIETO, M.D. (1993): The teacher as mediator in learning for gifted children. Abstracts of the Papers submited to the Third European Council for High Ability. Volume 1. Tooronto: Hoggrefe and Huber Publishers.

PORTER, L (1999) Gifted Young Children A Guide for Teachers and Parents. Open University Press

PUENTE FENERAS, A. (comp) (1994). Estilos de aprendizaje y enseñanza Madrid: CEPE.

PuRCELL, J.H. y RENZULLl,J.S. (1998). The Total Talent Portfolio: A systematic plan to identify and nuture gifts and talents. Mansfield Center, CT: Creative Learning Press.

RAVEN, J. C. (1970). Guide to using the coloure progressive matrices. En A. A. Anastasie, Test psicológicos. La Habana: Edición Revolucionaria

RENZULLL, J. (1981) What makes giftedness? Reexamining a Definition. En: Psichology and Education of the Gifted. Nueva York: Irvington Publishers.

RENZULLL J. S. (1978): What makes giftedness? Re - examining a definition. Phi Delta Kappan, 60, 180184.

Ruiz, C. y Ríos, P. (1994) Estrategias cognitivas. En A. Puente (Ed.) Estilos de aprendizaje y enseñanza. Madrid: CEPE.

SCHMECK, R.S (1988) Learning strategies and learning styles. Nueva York: Plenum Press.

SELMES, I (1987) La mejora de las habilidades para el estudio. Barcelona: Paidós

STEMBERG, RJ (1990). Inlellectual styles. Theory and classroom interaction. Washington National Educatión Asociation of the United States Research for better schools.

TANNEBAUn, A. J. (1983): Gifted children: Psychological and educational perspectives. New York: Macmillan.

VIGOTSKY, L. S. (1987) Historia del desarrollo de las funciones psíquicas superiores. La Habana: Ciencias Sociales. 
WHITMORE, J. (1986): Nuevos retos a los métodos de identificación habituales. En J. Freeman, (ed): Los niños superdotados. Aspectos psicológicos y pedagógicos. Madrid Santillana - Aula XXI. 


\title{
Contactar
}

Revista lberoamericana de Educación

\author{
Principal OEI
}

